\title{
CHARGE COLLECTION AND ELECTRODE STRUCTURES IN IONIZATION AND PHONON BASED DARK MATTER DETECTORS.
}

\author{
T. Shutt ${ }^{1}$, M.Kesden ${ }^{2}$, S.Golwala ${ }^{2}$, J.Emes $^{3}$, J. Hellmig $^{4}$, B. Sadoulet ${ }^{4}$, \\ B. A. Young ${ }^{5}$ and the CDMS detector group. \\ ${ }^{\prime}$ Department of Physics, Princeton University, Princeton, NJ 08544, USA \\ ${ }^{2}$ Department of Physics, California Institute of Technology, Pasadena, CA 91125, USA \\ ${ }^{3}$ Lawrence Berkeley National Laboratory, Berkeley, CA 94720, USA \\ ${ }^{4}$ Center for Particle Astrophysics, University of California, Berkeley, CA 94720, USA \\ ${ }^{5}$ Department of Physics, Santa Clara University, Santa Clara, CA 95053, USA
}

\begin{abstract}
We report on progress in our understanding of the physics of of implanted contacts, amorphous layer structures and Schottky contacts on detectors based on the simultaneous ionization and phonon based at $20 \mathrm{mK}$. Loss of charge for events near the contacts limits the capabilities of these detectors for detecting dark matter, an effect which is minimized by use of amorphous blocking layers.
\end{abstract}

\section{DEAD LAYER PROBLEM, DEVICES STUDIED}

The detectors used for CDMS are based on simultaneous ionization and phonon measurement which distinguishes electron and nuclear recoils based on the lower ionization yield of nuclear recoils. However, with the small charge-collection electric fields we use (typically below a few $\mathrm{V} / \mathrm{cm}$ ) charges from events near a contact can diffuse into the contact structure and be lost before being drifted across the detector and measured. This "dead layer" is of serious concern because it causes low energy external background electrons to be confused with the signal of nuclear recoils from dark matter. At the last LTD conference we reported on the use of amorphous blocking layers to solve this dead layer problem[1]. In this report we discuss in greater detail the physics of contacts at $20 \mathrm{mK}$, and also report on devices employing $\mathrm{Al}$ and $\mathrm{Au}$ Schottky structures.

The devices we studied, described in more detail previously[1], consist of p-type Ge bulk crystals, $1 \times 1 \mathrm{~cm}^{2}$, and 0.1 or $0.4 \mathrm{~cm}$ thick. An NTD Ge thermistor glued to each crystal measures the phonon energy. Charge collection as a function of depth was measured using the low energy photons from ${ }^{241} \mathrm{Am}$. $\mathrm{A}^{14} \mathrm{C}$ electron source was used to probe charge collection for low energy electrons. Several different chargecollection electrode structures were studied:

- "Standard", degenerately-doped implanted $\mathrm{p}^{+}$and $\mathrm{n}^{+}$contacts formed by $\mathrm{B}$ and $\mathrm{P}$ implantation (note that ${ }^{+}$indicates a degenerate contact, not the sign of charge).

- Amorphous layer structures[2]. An Al layer $\approx 2500 \AA$ thick deposited on hydrogenated amorphous Si layers with thicknesses between 175 and $275 \AA$.

CP605, Low Temperature Detectors, edited by F. S. Porter et al. (c) 2002 American Institute of Physics 0-7354-0049-0/02/\$19.00 513 
- Al (Schottky) contacts, deposited directly on the Ge either by sputtering or evaporation.

In these $20 \mathrm{mK}$ detectors, unlike in standard semiconductor ionization detectors, there is no thermally generated free charge from the impurity sites. Also, the detectors are operated in a situation in which these sites appear to be essentially all neutralized. Charge collection is very poor (especially in Si because of its low purity) until the detectors have been exposed to radiation with no bulk electric field present for sufficient time to create roughly as many electron-hole pairs as there are impurity sites in the crystal. The free charge generated by these events neutralizes the charged impurity sites that thermal equilibrium would otherwise maintain. In the absence of further of free charges from events, this non-equilibrium situation is stable. In this non-equilibrium situation, the concept of a Fermi level is not useful.

\section{FERMI LEVELS AND NEAR-CONTACT FIELDS}

The Fermi level should be well defined and constant throughout all the conductive parts of the contact structures and the external circuit. In the bulk, with no space charge, the band edges will be aligned with the band edges in the contacts, and we can make the following predictions. The Fermi levels on either side of the device are aligned if there is no external bias. If the contacts are identical, there is no electric field in the bulk. If the contacts are not identical, the band edges are tilted to line up the Fermi levels in the contacts. An external bias changes the relative position of the Fermi levels in the two contacts. In Figure 1 we illustrate this for $a p^{+}-p-n^{+}$device. In degenerate implanted contacts the Fermi levels are essentially at (to within a few $\mathrm{mV}$ ) the band edges. Thus in general there will be an electric field in the bulk (due to thin layers of space charge in the contact) unless the applied "offset" voltage is $E_{\text {gap }} / e$, or $\approx$ $0.75 \mathrm{~V}$ in Ge. At this bias one expects no bulk field and no charge collection. Experimentally, collection vanishes when a voltage of $\approx 0.73 \mathrm{~V}$ is applied to the $\mathrm{p}^{+}$ contact in such a device, confirming our expectation. In addition, events of only one polarity are observed for all other bias voltages. This is only true if there is no space charge in the bulk, since space charge creates curved bands which, for some external bias voltages, give regions in the detector with different electric field directions.

Devices with identical amorphous structures on both sides had small $(\leq 10 \mathrm{mV})$ offset voltages. However two devices with an implanted $\mathrm{p}^{+}$contact on one side and an amorphous structure on the other displayed "offset voltages" (applied to the $\mathrm{p}^{+}$ contact) at which charge collection vanishes of $0.35 \mathrm{~V}$ and $0.20 \mathrm{~V}$. The offset voltage measures the difference in Fermi level between in the amorphous contact and the $\mathrm{p}^{+}$contact (which is at the valence band edge). The difference in offset voltages between the devices suggests that surface states, presumably affected by the amorphous layer deposition conditions, are important. An Al-Ge-Al Schottky device had an offset voltage that was $\approx 0.23 \mathrm{~V}$ between the sputtered and evaporated $\mathrm{Al}$ contacts, again suggesting again the importance of surface states.

A further probe of the band structure and energy levels is provided by the total energy generated as phonons. An event depositing an energy $E_{d}$ creates 
$N_{q}=E_{d} / \varepsilon$ electron-hole pairs (in Ge, $\varepsilon=3.0 \mathrm{eV}$ ). As the charges drift in the bulk electric field they dissipate energy as phonons. At the contacts they relax to the Fermi level. For the bias voltage shown in Figure 1, the charges are already at the Fermi level and the energy released is negligible. But if the bias voltage is increased to just above $E_{g a p} / e$ where $E_{g a p}$ is the band gap energy, the direction of charge drift reverses, and electrons reaching the $\mathrm{p}^{+}$contact relax across the band, as do holes reaching the $\mathrm{n}^{+}$ contact. Thus the measured phonon energy changes by twice the band gap per charge pair as voltage is varied from just above to just below $E_{\text {gap }} / e$. In Figure 2 we show the measured energy for a $\mathrm{p}^{+}-\mathrm{p}-\mathrm{n}^{+}$device and $60 \mathrm{keV}$ photons. As expected, a jump in phonon energy of $2 N_{q} E_{\text {gap }} \approx 30 \mathrm{keV}$ is observed at a bias of $E_{\text {gap }} / e=0.75 \mathrm{~V}$.

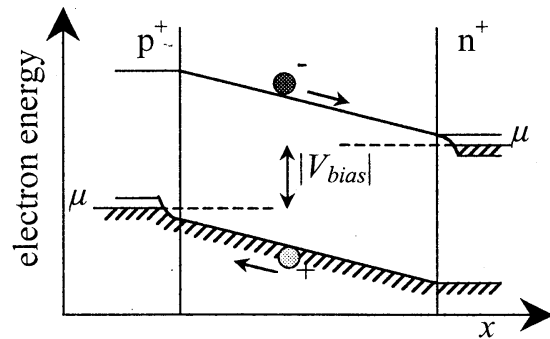

FIGURE 1. Energy bands in a $\mathrm{p}^{+}-\mathrm{p}-\mathrm{n}^{+}$device with an applied bias voltage. For the bias shown, electrons (dark circles) are collected at the $n^{+}$ contact and holes at the $\mathrm{p}^{+}$contact.

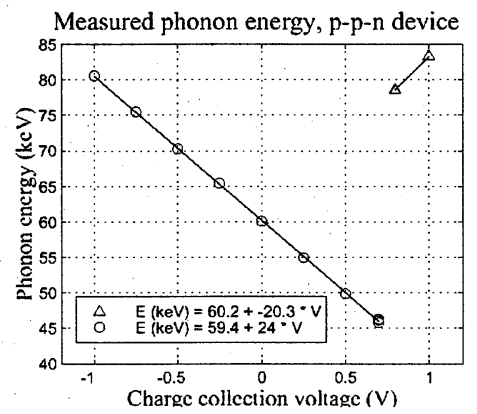

FIGURE 2. Phonon energy for $60 \mathrm{keV}$ photons in a $\mathrm{p}^{+}-\mathrm{p}-\mathrm{n}^{+}$device versus applied bias voltage.

Another phenomenon of both practical and theoretical importance is near-contact fields created by a layer of space charge. In thermal equilibrium such structures occur whenever the Fermi-level in a contact is not aligned with the Fermi level in the bulk, as for instance at a $\mathrm{n}^{+}-\mathrm{p}$ "diode". Such fields exist are small or non-existent near all implanted contacts, including $\mathrm{n}^{+}$-p diodes, indicating a remarkable degree of neutrality near the contact. They were largely absent in the amorphous layer devices. In the Schottky devices, for electric fields that push electrons away from the contact a strong near-contact field opposed the external field, yielding very poor charge collection. For the opposite bias direction the near-contact field was small or absent. We have yet not developed a comprehensive understanding of these varied phenomena.

\section{CHARGE COLLECTION, AMORPHOUS LAYER DEVICES}

The dead-layer performance of devices has been probed using events from individual lines from ${ }^{241} \mathrm{Am}$ (primarily 18 and $21 \mathrm{keV}$, which penetrate 30 and $50 \mu \mathrm{m}$ in $\mathrm{Ge}$ ) to derive the fraction charge collection versus depth, $q(x)$. We have also modeled the diffusion process leading to charge loss. The electrons lose energy at 20 $\mathrm{mK}$ only by phonon emission, a process first described by Abakumov[3,4]. The contact is treated simply as a surface that either reflects the charges with an energy independent probability $R$, or absorbs them. A typical measured and modeled curve of 


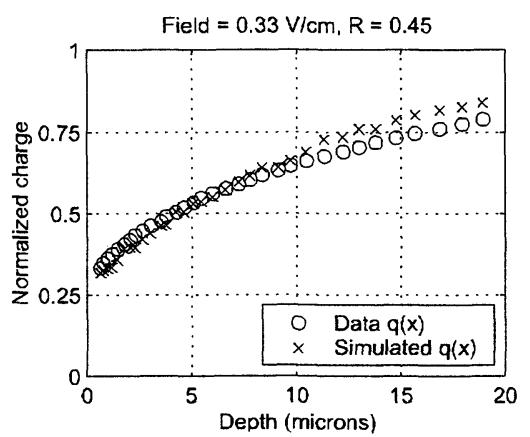

FIGURE 3. Comparison of measured and calculated charge collection for events near a ${ }^{+}$implanted contact with a $5 \mathrm{~V} / \mathrm{cm}$ applied field.

$q(x)$ is shown in Figure 3 for a $\mathrm{p}^{+}$implanted contact. Reasonable agreement between the model and data was obtained for all biases with a single value of $R$ for each bias polarity. For this device charge collection at the surface is $30 \%$ of full, which is the same as nuclear recoils. These therefore contacts are a poor choice for dark matter detectors.

However amorphous blocking layer contacts largely solve this dead layer problem. At the surface charge collection is $\geq 50 \%$, even for very low bias. Measurements with low energy electrons show that better than $95 \%$ are distinguished from nuclear recoils for energies above $\approx 20 \mathrm{keV}$. A qualitative explanation is that the amorphous layer, with an effective band gap larger than Ge, serves as a blocking layer for charges, decreasing the likelihood that charges will be lost in the contact before being pulled away by the applied field. The amorphous layer is slightly conductive, so that the charge species that should be collected at the amorphous contact eventually trickles through.

In conclusion, semiconductor contacts at $20 \mathrm{mK}$ exhibit a number of interesting features not seen in standard detectors because of the lack of thermally generated free charge. We have explored a number of these phenomena, and finally demonstrated that amorphous blocking layers represent at least a partial solution to the dead layer problem. Further understanding of the physics of the contacts might lead to further improvements in device performance.

\section{ACKNOWLEDGMENTS}

The experimental aspects of this work were supported by the Center for Particle Astrophysics, a NSF Science and Technology Center operated by the University of California, Berkeley, under Cooperative Agreement No. AST-91-20005, by the National Science Foundation under Grant No. PHY-9722414, and by the Department of Energy under contracts DE-AC03-76SF00098, DE-FG03-90ER40569, and DEFG03-91ER40618.

\section{REFERENCES}

1. Shutt, T., etal., "A Solution to the Dead Layer Problem in Ionization and Phonon-Based Dark Matter Detectors", Proceedings of the VIII International Workshop on Low Temperature Detectors, 1999.

2. For a general reference on semiconductor contacts, albeit well above $20 \mathrm{mK}$, see S. M. Sze, Physics of Semiconductor Devices, New York: John Wiley \& Sons, 1981.

3. See, for example, Luke, P., Rossington C.S., and Wesela M.F., IEEE Trans. Nucl. Sci. 41, 4, 1074 (1994).

4. Abakumov, V.N., Sov. Phys. Semicond. 12, 1 (1978). 